\title{
Considerations for the use of plasma cytokeratin 18 as a biomarker in pancreatic cancer
}

\author{
C Dive', RA Smith², E Garner², T Ward', S St George-Smith', F Campbell ${ }^{3}$, W Greenhalf', P Ghaneh ${ }^{2}$ \\ and JP Neoptolemos ${ }^{*}, 2$
}

'Clinical and Experimental Pharmacology Group, Paterson Institute for Cancer Research, University of Manchester, Manchester, UK; ${ }^{2}$ Liverpool Experimental Cancer Medicines Centre and NIHR Pancreas Biomedical Research Unit, Royal Liverpool University Hospital, 5th Floor UCD Building, Daulby St, Liverpool L69 3GA, UK; ${ }^{3}$ Department of Pathology, Royal Liverpool University Hospital, Prescot St, Liverpool L7 8XP, UK

\begin{abstract}
BACKGROUND: Enzyme-linked immunoassays of full-length (M65) and/or caspase-cleaved (M30) cytokeratin I8 (CKI8) released from epithelial cells undergoing necrosis and/or apoptosis, respectively, may have prognostic or predictive biomarker utility in a range of solid tumour types. Characterisation of baseline levels of circulating full length and cleaved CKI 8 specifically in patients with pancreatic cancer.

METHODS: Plasma samples from 103 patients with pancreatic cancer stored at $-80^{\circ} \mathrm{C}$ were assayed for M65 and M30 levels. The median (inter-quartile range (IQR)) duration of plasma storage was $34(23-57)$ months. Patients with metastatic disease $(n=19)$ were found to have greater median (IQR) M65 levels $\left(I \mid 45(739-1698) \cup I^{-1}\right)$ compared with the locally advanced $(n=20 ; 748$ $\left.(406-|| 50) \cup I^{-1}\right)$ and resected $\left(n=64 ; 6 I 2(33 \mid-987) \cup I^{-1}\right)$ patients $(P=0.002)$. Elevated M65 levels were associated with poorer overall survival on univariate $(P<0.00 \mathrm{I})$ but not multivariate $(P=0.202)$ analysis. M65 concentrations also exhibited significant associations with concurrent serum-bilirubin levels $(P<0.00 \mathrm{I})$ and the duration of plasma storage $(P<0.00 \mathrm{I})$.

CONCLUSIONS: Baseline plasma CKI 8 levels in pancreatic cancer are affected by the presence of obstructive jaundice and prolonged plasma storage. Clinical biomarker studies utilising serial CKI 8 levels are warranted in pancreatic cancer, provided consideration is given to these potentially confounding factors.

British Journal of Cancer (2010) I 02, 577-582. doi:I0.1038/sj.bjc.6605494 www.bjcancer.com

Published online 5 January 2010

(C) 2010 Cancer Research UK
\end{abstract}

Keywords: pancreatic cancer; cytokeratin 18; circulating biomarker; M65; M30; necrosis; apoptosis

Pancreatic ductal adenocarcinoma is a leading cause of cancer deaths (http://www-dep.iarc.fr/) wherein, because of its characteristically late presentation, only $10-15 \%$ of patients present with resectable tumours (Alexakis et al, 2004). For the remaining $85-90 \%$ of patients presenting with inoperable disease, administration of systemic chemotherapy represents the most widely utilised palliative treatment modality (Yip et al, 2006). Conventional chemotherapy regimens incorporating either 5-FU or gemcitabine typically result in only modest improvement in overall survival (Sultana et al, 2007). One of the key challenges in accelerating improved cancer therapeutics is the identification of biomarkers to enable early objective assessment of tumour responses to chemotherapy (Kummar et al, 2007).

Necrosis of malignant and normal epithelial cells cause release of cytokeratin 18 (CK18) (Kramer et al, 2004), a type I intermediate filament protein and a component of the intracellular cytoskeleton (Fuchs and Weber, 1994). Caspase-mediated cleavage of the CK18 contributes to the degradation of the intracellular cytoskeleton if

* Correspondence: Professor JP Neoptolemos, The Owen and Ellen Evans Chair of Cancer Studies, Liverpool Cancer Research UK Centre, Royal Liverpool University Hospital, 5th Floor UCD Building, Daulby St, Liverpool L69 3GA, UK; E-mail: j.p.neoptolemos@liverpool.ac.uk

Received 6 October 2009; revised 16 November 2009; accepted 20 November 2009; published online 5 January 2010 epithelial cells undergo apoptosis (Leers et al, 1999). Enzymelinked immunosorbent assays (ELISAs) have been developed to measure circulating concentrations of both full-length CK18 (M65) and caspase-cleaved CK18 fragments (M30) (Kramer et al, 2004). Thus, the M65 ELISA reports necrotic and apoptotic epithelial cell death, whereas the M30 ELISA reports levels of epithelial apoptosis specifically (Kramer et al, 2004). Early studies suggest that these assays may have important clinical biomarker utility, as increased levels of circulating CK18 may be prognostic and/or predict tumour response to chemotherapy in a number of different solid tumours (Steele et al, 2008; Scott et al, 2009). Studies on specific tumour types include lung (Ulukaya et al, 2007; Hou et al, 2009), breast (Olofsson et al, 2007), prostate (Kramer et al, 2006), head and neck (Ozturk et al, 2009), colorectal (Ausch et al, 2009; Koelink et al, 2009) and testicular (de Haas et al, 2008) tumours. Although a recent study concluded that plasma levels of CK18 are a potential marker of tumour response in patients with advanced gastrointestinal malignancy (Scott et al, 2009) no previous studies have been reported in patients with pancreatic cancer.

The objective of this study was to characterise the baseline levels of circulating plasma CK18 concentrations using both the M65 and M30 ELISA assays in patients with pancreatic cancer. Correlations were also examined between CK18 and the circulating levels of carbohydrate antigen 19-9 (CA19-9), the only widely used biomarker in pancreatic cancer (Smith et al, 2008). Preoperative 
biliary stenting and concurrent liver function were also investigated in the interpretation of CK18 levels, as obstructive jaundice has been shown to be a confounding factor in proteomic studies (Yan et al, 2009) along with effect of plasma storage duration (Cummings et al, 2007; Greystoke et al, 2008).

\section{MATERIALS AND METHODS}

\section{Patients and plasma collection}

A total of 103 non-consecutive pancreatic adenocarcinoma patients with stored samples, treated at the Royal Liverpool University Hospital between 1997 and 2007 were identified from a prospective clinical database. All patients gave written consent for blood, tissue and clinical data to be used for research purposes.

All patients with obstructive jaundice routinely underwent biliary stenting at endoscopic retrograde cholangio-pancreatography. If endoscopic biliary decompression was unsuccessful, patients underwent percutaneous transhepatic cholangiography or combined procedures in order to decompress the biliary tree. In all, 64 patients then underwent a surgical resection for histologically confirmed pancreatic ductal adenocarcinoma (35 men and 29 women) with a median age of 68 (inter-quartile range $(I Q R)=61-73)$ years. A total of 39 patients had advanced pancreatic adenocarcinoma (22 men and 17 women) with a median age of $68(\mathrm{IQR}=58-77)$ years. The diagnosis of advanced disease was made at laparotomy in 30 of $39(77 \%)$ patients. The remaining 9 cases $(26 \%)$ were diagnosed as unresectable on initial imaging or at staging laparoscopy. In all, 15 of 64 patients undergoing resection went on to receive adjuvant therapy, chemotherapy in 13 and chemoradiotherapy in two. Patients with advanced cancer were managed at multiple referral institutions and data relating to palliative chemotherapy were available for 22 patients, 8 of whom had undergone chemotherapy. There was no significant change of the adjuvant or palliative therapy protocols during the study period.

Plasma was collected on the day before surgical intervention and stored at $-80^{\circ} \mathrm{C}$. Plasma was collected at the time of diagnosis in the nine patients managed nonoperatively. The median time interval for plasma storage (from the date of plasma collection to the date of assay) was 34.1 ( $\mathrm{IQR}=22.7-57.0$ ) months. Preoperative serum CA19-9 and concurrent bilirubin levels were also retrieved, along with details regarding resected histopathological tumour characteristics and patient survival data. Overall survival times were calculated from the date of plasma sampling to the date of death.

\section{Detection of M65 and M30 antigens}

The M65 and M30 (Apoptosense) ELISA kits (Peviva, Ströskarlsvägen, Sweden) were used for the plasma biomarker analyses according to previously described methods and according to good clinical laboratory practice (GCLP, Cummings et al, 2005). An apoptotic index was calculated from the ratio of caspase-cleaved CK18 as a proportion of overall CK18 (M30 divided by M65) as previously described (Kramer et al, 2004).

\section{Preparation of tissue microarray (TMA)}

The original haematoxylin and eosin (H\&E) stained histopathology slides from resected cases were retrieved and reviewed by a consultant pathologist (FC). Slides were marked in order to target cancer cores for subsequent sampling from stored paraffinembedded tissue blocks. It was ensured that targeted areas were away from resection margins to avoid diathermied tissue. Duplicate cores taken from three areas within each tissue block were sampled for each patient. Normal tissue from kidney, colon and duodenum were used for orientation cores. Five control blocks from chronic pancreatitis specimens were also used in the TMA.

The marked slide and paraffin-embedded tissue block were aligned so that the targeted area of tumour on the donor block could be determined. Using a manual tissue arrayer (Beecher Instruments, Sun Prairie, WI, USA), $0.6 \mathrm{~mm}$ diameter tissue cores were taken to a depth of $4 \mathrm{~mm}$ from the targeted area of tumour in the donor block. The tissue cores were placed into the recipient paraffin block $1 \mathrm{~mm}$ apart. The TMA blocks were cut using a Leica Reichert-Jung BIOCUT 2035 microtome (Leica Biosystems, Wetzlar, Germany) in $5 \mu \mathrm{m}$ sections. Two $5 \mu \mathrm{m}$ sections were cut

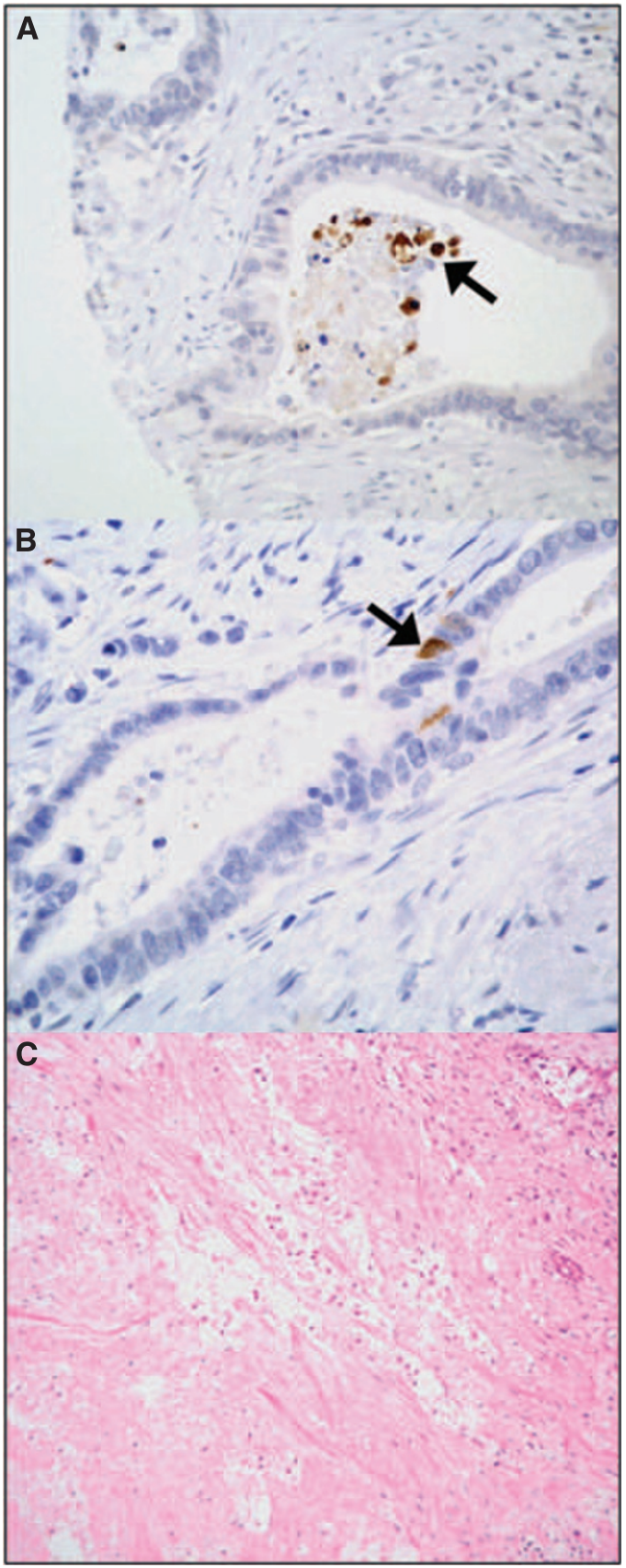

Figure I (A) Example of shed cells within a cancer gland exhibiting positive staining for activated caspase-3 on immunohistochemistry (arrowed) in a core of pancreatic ductal adenocarcinoma. (B) Example of sporadic nature of activated caspase-3 staining within the malignant ductal epithelium. (C) Haematoxylin and eosin (H\&E) stained slide of pancreatic ductal adenocarcinoma exhibiting widespread tumour necrosis. 
from each block and mounted on X-tra adhesive Surgipath slides in preparation for immunohistochemistry (IHC).

\section{Detection of cleaved caspase 3}

Cleaved caspase 3 was assessed by immunohistochemistry using a validated method to GCLP. Tissue microarray sections were deparaffinised, re-dehydrated and antigen retrieval was carried out using microwaves for $25 \mathrm{~min}$ in citric acid buffer $(10 \mathrm{~mm}, \mathrm{pH}$ 6.0). Sections were then blocked by immersing in a $0.3 \%$ hydrogen peroxide solution for $30 \mathrm{~min}$ to remove exogenous and endogenous peroxidase. The slides were blocked using $10 \%$ casein solution for $1 \mathrm{~h}$ to remove background staining. Slides were then incubated overnight with the primary antibody (Cell Signaling Technology, Boston, MA, USA; ref: \#9661S) at $4{ }^{\circ} \mathrm{C}$ in a humidified tray. Slides were then washed in PBS and goat anti-rabbit (Vectastain ABC kit from Vector Laboratories, Burlingame, CA, USA; ref: PK-4001) secondary antibody was added for $30 \mathrm{~min}$. The slides were washed with PBS and ABC kit (Envision kit, Vector Laboratories) was applied (according to manufacturer's instructions). Sections were washed in PBS before visualization of signal using DAB reagent. Sections were then counterstained with haematoxylin before mounting.

Table I Demographics and clinicopathological data

\begin{tabular}{|c|c|}
\hline Total number of patients & 103 \\
\hline Median age (IQR) (years) & $68(60-74)$ \\
\hline Male: Female & $57: 46$ \\
\hline Resected cases: & 64 \\
\hline Median tumour size (IQR) (mm) & $30(22-38)$ \\
\hline $\mathrm{TI} / \mathrm{T} 2: \mathrm{T} 3 / \mathrm{T} 4$ & $11: 52$ \\
\hline $\mathrm{NO}: \mathrm{NI}$ & $10: 52$ \\
\hline Well/moderate/poor differentiation & $10 / 35 / 18$ \\
\hline $\mathrm{RO}: \mathrm{RI}$ & $19: 45$ \\
\hline Unresected cases: & 39 \\
\hline Locally advanced & 20 \\
\hline Metastatic & 19 \\
\hline Median M65 (IQR) $\left(\mathrm{UI}^{-1}\right)$ & $724(4|1-1| 88)$ \\
\hline Median M30 (IQR) $\left(\mathrm{UI}^{-1}\right)$ & $212(149-327)$ \\
\hline Median M30: M65 ratio (IQR) & $0.32(0.24-0.49)$ \\
\hline Median preoperative CAI9-9 (IQR) $\left(\mathrm{kUI}^{-1}\right)$ & $463(151-1265)$ \\
\hline Median preoperative bilirubin (IQR) $\left(\mu \mathrm{mol} \mathrm{I}^{-1}\right)$ & $23(12-57)$ \\
\hline Median duration of plasma storage (IQR) (months) & $34(23-57)$ \\
\hline
\end{tabular}

$\mathrm{CA}=$ carbohydrate antigen; $\mathrm{IQR}=$ interquartile range. The median $(\mathrm{IOR})$ time interval from the date of preoperative CAI9-9 estimation to the date of plasma sampling was $28(19,38)$ days. The median $(\mathrm{IQR})$ time interval for liver function tests was I (I, 2) day. Histological data was incomplete for one patient. Preoperative CA19-9 and bilirubin data were incomplete for 11 and 14 patients, respectively. The median (IQR) values for M30 and M65 in 82 healthy controls were 198 $(|55,3| 7) \cup I^{-1}$ and $240(19 \mid, 339) \cup I^{-1}$, respectively (Greystoke et al, 2008).

\section{Immunohistochemical scoring}

The activated caspase- 3 immunohistochemistry was scored by a consultant pathologist (FC) according to the presence of positive staining in the intraluminal shed cells within cancer glands (Figure 1A). Only tissue cores exhibiting one or more shed cells were scored. Cases were classified as either positive or negative based on the proportion of positively stained cells present. Cases where more than $50 \%$ of shed cells exhibited positive staining in a majority of cancer cores were classified as positive. Owing to the infrequent staining exhibited in the intact malignant epithelium (Figure 1B), no attempt to score the epithelial staining was made.

The original H\&E stained histopathology slides for the resected pancreatic specimens were also evaluated by the same consultant pathologist (FC) to make a quantitative assessment of tumour necrosis on microscopic examination. Cases were allocated a percentage score $(0,1,5,10,25$ or $50 \%)$ according to the proportional area of tumour material exhibiting microscopic features of necrosis (Figure 1C). Areas of fibrosis, sclerosis and autolysis on microscopy were not included as part of this assessment. The pathologist was blinded to the ELISA results and survival data during scoring of both the activated caspase- 3 immunohistochemistry and the microscopic assessment of tumour necrosis.

\section{Statistical analysis}

Continuous data were described using median values, IQR and 95\% confidence intervals (CIs) with Mann-Whitney U-testing or Kruskal-Wallis testing for non-parametric comparisons of continuous data. Categorical data were analyzed using the $\chi^{2}$ test. Spearman's rank correlation was used to analyse the relationship between two individual continuous variables. CA19-9 was normalised for regression analyses by logarithmic transformation ( $\left.\log _{\mathrm{e}} \mathrm{CA19}-9\right)$. Survival data were analysed using the KaplanMeier method with log-rank testing for the comparison of survival curves. Cox's proportional hazards regression was used for multivariate analysis. Prognostic variables of univariate significance were selected for inclusion in the multivariate model. A $P$-value of 0.05 was considered significant.

\section{RESULTS}

Of the 103 patients analysed, plasma M65 levels were measureable for 102 and M30 levels measureable for 98 patients. Both assays were measureable for 97 patients. Table 1 gives details of the demographic and clinicopathological data for the overall patient group. The median (IQR) plasma M65 level recorded in the overall patient group was $724(411,1188) \mathrm{Ul}^{-1}$, whereas the median plasma M30 level was $212(149,327) \mathrm{Ul}^{-1}$ and the M30: M65 ratio was $0.32(0.24,0.49)$. The median (IQR) reference values for M30 and M65 were determined in 82 healthy controls (30 men and 52 women) with a median age of $45(35,55)$ years and were 198

Table 2 Parameters according to resectability and presence of metastases

\begin{tabular}{|c|c|c|c|c|}
\hline & $\begin{array}{l}\text { Resected cases } \\
(N=64)\end{array}$ & $\begin{array}{l}\text { Locally advanced } \\
\text { unresected cases }(N=20)\end{array}$ & $\begin{array}{l}\text { Metastatic unresected } \\
\text { cases }(N=19)\end{array}$ & $P$-value \\
\hline Median age (IQR) (years) & $68(6 \mid-73)$ & $70(64-77)$ & $65(57-75)$ & 0.406 \\
\hline Male: Female & $35: 29$ & $8: 12$ & $14: 5$ & $0.105 *$ \\
\hline Median M65 (IQR) $\left(\mathrm{UI}^{-1}\right)$ & $611(331-987)$ & $748(406-1150)$ & | $445(739-1698)$ & 0.002 \\
\hline Median M30 (IQR) $\left(\mathrm{UI}^{-1}\right)$ & $192(|47-3| 1)$ & $215(142-348)$ & $324(224-453)$ & 0.058 \\
\hline Median M30: M65 ratio (IQR) & $0.39(0.24-0.50)$ & $0.29(0.21-0.40)$ & $0.28(0.25-0.33)$ & 0.184 \\
\hline Median preoperative CAI9-9 (IQR) $\left(\mathrm{kUI}^{-1}\right)$ & $356(81-893)$ & $416(217-2310)$ & $1628(58|-33| 2)$ & 0.007 \\
\hline Median preoperative bilirubin (IQR) $\left(\mu \mathrm{moll}^{-1}\right)$ & $22(12-37)$ & $23(||-5 \mid)$ & $99(21-204)$ & 0.004 \\
\hline Median duration of plasma storage (IQR) (months) & $29(21-42)$ & $43(29-60)$ & $106(32-114)$ & 0.003 \\
\hline
\end{tabular}

$\mathrm{CA}=$ carbohydrate antigen; $\mathrm{IQR}=$ inter quartile range. Quoted $P$-values for Kruskal-Wallis test. * $\chi^{2} P$-value. 
$(155,317) \mathrm{Ul}^{-1}$ and $240(191,339) \mathrm{Ul}^{-1}$, respectively (Greystoke et al, 2008). The three clinical groups of resected, locally advanced not resected and metastasis groups may be considered as demonstrating increasing tumour burden and so the results for the M30 and M65 levels and the M30:M65 ratios are shown by these groups in Table 2 . The median (IQR) M65 levels significantly increased with increasing degrees of tumour burden being 1145 $(572,1479) \mathrm{Ul}^{-1}$ for patients with metastatic disease compared with $748(406,1150) \mathrm{Ul}^{-1}$ in patients with locally advanced nonresectable disease and $612(331,987) \mathrm{Ul}^{-1}$ in patients who had resection (Kruskal-Wallis, $P=0.002$; Table 2 and Figure 2).

\section{Confounding factors}

Carbohydrate antigen $19-9(P=0.007)$ and bilirubin $(P=0.004)$ levels were also associated with the increasing tumour burden (Table 2). Plasma M65 levels were found to exhibit a strong correlation with concurrent serum-bilirubin levels (Spearman, $\rho=0.439, P<0.001$ ) but not CA19-9 levels. A significant correlation between M65 levels and the duration of plasma storage overall was found (Spearman, $\rho=0.487, P<0.001$ ) and the period of

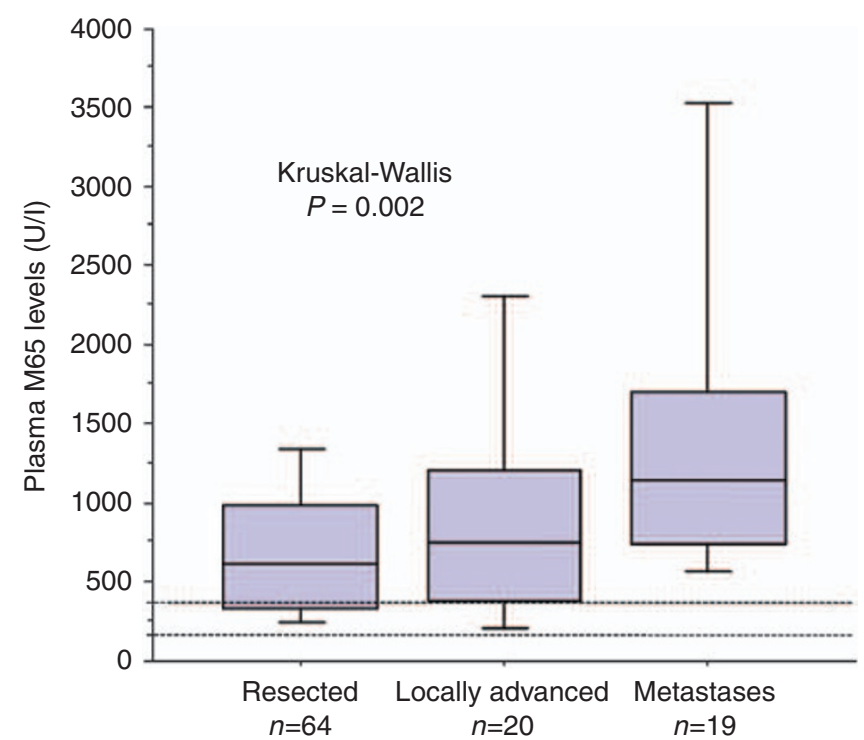

Figure 2 Box plot illustrating the effect of tumour resectability and presence of metastasis on plasma M65 levels. The dotted lines represent the inter quartile range (IQR) reference values of 191 and $339 \mathrm{UI}^{-1}$ for healthy controls (Greystoke et al, 2008). plasma storage was also unexpectedly found to be significantly longer in the group with metastases $(P=0.007)$.

\section{Plasma CK18 levels and survival}

Resectability, the presence of metastases and M65, Ca19-9 and bilirubin levels were all significantly associated with survival (all $P<0.001$ ) on univariate analyses (Table 3 and Figure 3 ). Resected patients had a median survival of $13.9(95 \% \mathrm{CI}=11.0-16.4)$ months compared with $7.3(95 \% \mathrm{CI}=5.5-11.0)$ months for locally advanced tumours and $2.5(95 \% \mathrm{CI}=1.2-3.6)$ months for patients with metastatic disease (log rank, $P<0.001$ ). Increasingly elevated plasma M65 levels were associated with poorer survival in the overall patient group on univariate analysis both as a continuous (Cox, $P<0.001$; Table 3 ) and dichotomised variable (log rank, $P=0.007$; Figure 3B). Neither M30 levels (Cox, $P=0.052)$ nor the M30:M65 ratio (Cox, $P=0.096$ ) were significant univariate prognostic variables in the overall patient group. The results of a multivariate analysis including M65 levels, tumour resectability, CA19-9 and bilirubin are shown in Table 3. In this model the only independent significant prognostic factors were resectability and the presence of metastases (both $P<0.001$ ).

\section{Histopathological assessment of necrosis and apoptosis in resected cases}

There was no significant relationship between plasma CK18 levels and tumour histology. The original histopathology slides with corresponding paraffin-embedded tumour material were available in 58 of 64 resected patients (91\%). A total of 17 patients $(29 \%)$ exhibited significant areas of tumour necrosis (i.e. $\geqslant 5 \%$ ) on microscopic assessment. There was no significant correlation between plasma M65 levels and the histological percentage score for microscopic tumour necrosis (Spearman, $\rho=0.084, P=0.531$ ). After the preparation of TMA, a total of 53 out of 58 cases had one or more evaluable cores containing cancer. In all, 45 cases exhibited shed cells, which could be evaluated for activated caspase-3 staining. Of these 45 cases, 25 (56\%) had positive staining and $20(44 \%)$ were negative. There was no significant difference in the median plasma M30: M65 ratio between positive $(0.37(\mathrm{IQR}=0.26-0.53))$ and negative cases $(0.36(\mathrm{IQR}=0.26-$ $0.48)$ ) for activated caspase-3 immunostaining (Mann-Whitney, $P=0.478)$.

\section{DISCUSSION}

Cytokeratin 18 is a type I intermediate filament protein component of the intracellular cytoskeleton in epithelial cells (Fuchs and

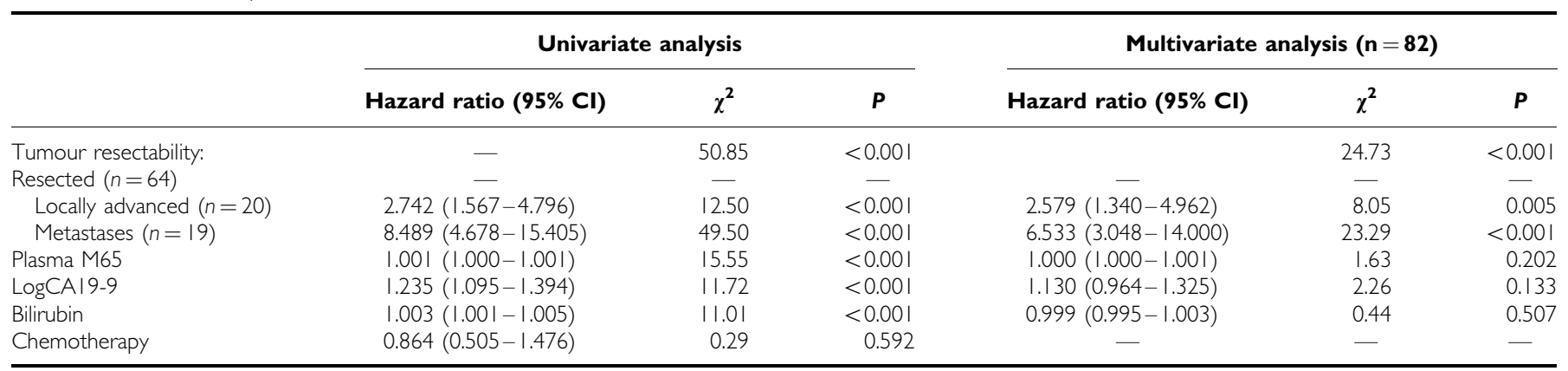

$\mathrm{CA}=$ carbohydrate antigen; $\mathrm{Cl}=$ confidence interval. *Plasma M65 levels, CA19-9 and bilirubin were modelled as continuous prognostic covariates. Quoted hazard ratios for continuous variables signify the relative hazard associated with each incremental increase in the covariate value by I U. Logarithmic transformation of CAI9-9 results was undertaken to normalise for Cox's regression because of the wide range of preoperative CAI9-9 results recorded in the overall patient group (from I-90 000 $\mathrm{kUI}^{-1}$ ). 


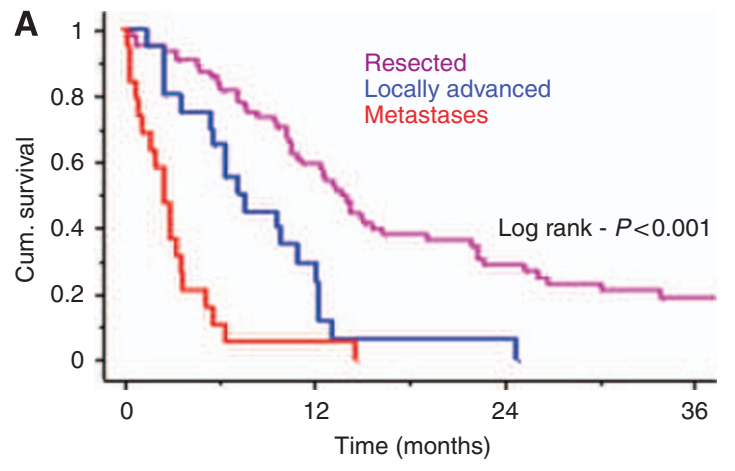

$\begin{array}{rcccc}\text { Resected } & 64 & 36 & 15 & 7 \\ \text { Locally advanced } & 20 & 5 & 1 & 0 \\ \text { Metastases } & 19 & 1 & 0 & 0\end{array}$

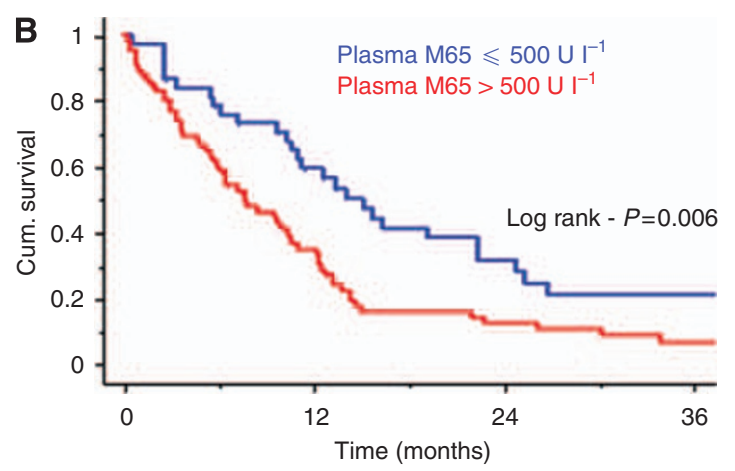

$\begin{array}{lllll}M 65 \leqslant 500 U^{-1} & 37 & 19 & 9 & 4 \\ M 65>500 U^{-1} & 65 & 22 & 7 & 3\end{array}$

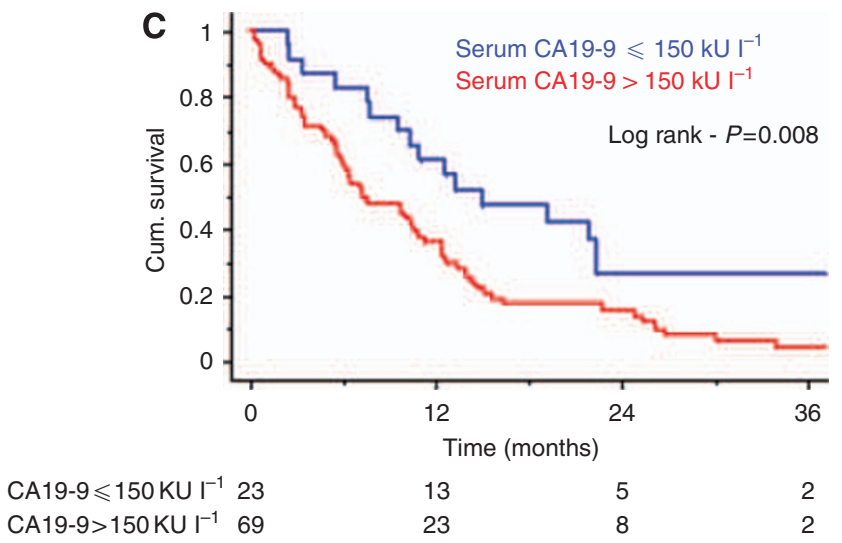

Figure 3 Kaplan-Meier curves to illustrate survival trends in overall patient group. (A) The expected pattern of worsening survival associated with more advanced disease was observed. The survival curves according to plasma M65 levels (B) were comparable with those seen for serum carbohydrate antigen (CA) 19-9 levels (C) in the overall patient group.

Weber, 1994). Increasing evidence exists to suggest that cytokeratins have an important role during epithelial apoptosis. Degradation of type I cytokeratins (including CK18) by activated caspases 3, 7 and 9 with consequent loss of mechanical integrity of the cell occurs during the execution phase of apoptosis to facilitate cell shrinkage and formation of apoptotic bodies (Leers et al, 1999; Schutte et al, 2004). During this process, CK18 is cleaved at two specific sites along the non-helical linker region of the protein (Asp238 and Asp396) exposing the Asp396 neo-epitope with the subsequent release of cleaved cytokeratin fragments into the extracellular space. Detection of this Asp396 neo-epitope by the
M30 ELISA is, therefore, only possible following caspasedependent apoptosis. It has been postulated that necrosis of epithelial cells releases full-length cytokeratins into the extracellular space following breakdown of the cell membrane (Bodenmüller et al, 1994). In vitro studies have shown that induction of necrosis in a human breast epithelial cell line is associated with a significant increase in the concentration of extracellular CK18, as recorded by the M65 ELISA (Kramer et al, 2004).

This study represents the first attempt to characterise circulating levels of CK18 in patients with pancreatic cancer in order to assess its clinical biomarker potential. The results from the M30 and M65 ELISA assays showed significant variability between patients with regard to the baseline levels of both full-length CK18 (M65) and the proportion of caspase-cleaved CK18 (M30:M65). Circulating total CK18 concentrations in this study were relatively high compared with prostate (Kramer et al, 2006) and breast (Olofsson et al, 2007) cancer and comparable with those of other gastrointestinal malignancies (Scott et al, 2009) and non-small-cell lung cancer (Hou et al, 2009). In keeping with the other malignant tumour types (Ulukaya et al, 2007; Hou et al, 2009; Koelink et al, 2009), elevated CK18 levels were associated with poorer survival in the overall patient group on univariate analysis, but in this series failed to reach significance on multivariate analysis.

There was no significant association between plasma M65 levels and the histopathological assessment of tumour necrosis. This finding may implicate additional factors other than intrinsic tumour biology having an important confounding effect on circulating M65 concentrations. A marked correlation was seen between the concurrent bilirubin levels and circulating CK18 levels. This observation is likely to be explained on the basis that obstruction of the main bile duct with consequent dilatation and epithelial disruption directly influences the balance of proliferation and cell death within the biliary epithelium (Lesage et al, 2001; Alpini et al, 2003). Low-grade cholangitis is quite common following biliary stenting and may also represent an additional confounding factor, as both generalised sepsis (Roth et al, 2004) and cholangitis (Yagmur et al, 2007) raise circulating CK18 concentrations. Other studies have demonstrated significant disturbances in circulating CK18 in patients with chronic liver disease (Hetz et al, 2007; Yagmur et al, 2007).

Information on the long-term antigen stability of the M30 and M65 ELISAs in stored human plasma is limited. A previous study (Cummings et al, 2007) in 20 patients with cancer showed no significant degradation of the M65 antigen after 2 years of storage at $-80^{\circ} \mathrm{C}$ although the $\mathrm{M} 30$ antigen exhibited increased values with extended storage in a proportion of patients and confirmed in a more recent study (Greystoke et al, 2008). The results from this, much larger, study have shown that antigen levels exhibit a trend towards more elevated values when stored over a longer period.

Appropriate pre-clinical validation of potential cancer biomarkers is essential before their utilisation in either routine clinical practice or trial settings (Cummings et al, 2008). This study highlights the fact that the pathophysiology of pancreatic cancer presents a number of different challenges with regard to the analysis of blood-borne biomarkers. Studies utilising serial CK18 measurements to determine tumour responses to cytotoxic therapy in pancreatic cancer should give adequate consideration to the potential confounding factors of concurrent obstructive jaundice and the duration of sample storage.

\section{ACKNOWLEDGEMENTS}

The authors thank Mr Martin Greaves for his technical assistance in IHC. This study was supported by Cancer Research UK (Registered Charity No. 1089464) and the National Institute for Health Research. 


\section{REFERENCES}

Alexakis N, Halloran C, Raraty M, Ghaneh P, Sutton R, Neoptolemos JP (2004) Current standards of surgery for pancreatic cancer. Br J Surg 91: $1410-1427$

Alpini G, Ueno Y, Tadlock L, Glaser SS, LeSage G, Francis H, Taffetani S, Marzioni M, Alvaro D, Patel T (2003) Increased susceptibility of cholangiocytes to tumor necrosis factor-alpha cytotoxicity after bile duct ligation. Am J Physiol Cell Physiol 285: 183-194

Ausch C, Buxhofer-Ausch V, Olszewski U, Hinterberger W, Ogris E, Schiessel R, Hamilton G (2009) Caspase-cleaved cytokeratin 18 fragment (M30) as marker of postoperative residual tumor load in colon cancer patients. Eur J Surg Oncol 35: 1164-1168

Bodenmüller H, Ofenloch-Hahnle B, Lane EB, Dessauer A, Bottger V, Donie F (1994) Lung cancer-associated keratin 19 fragments: development and biochemical characterisation of the new serum assay Enzymun-Test CY-FRA 21-1. Int J Biol Markers 9: 75-81

Cummings J, Ward TH, LaCasse E, Lefebvre C, St-Jean M, Durkin J, Ranson M, Dive C (2005) Validation of pharmacodynamic assays to evaluate the clinical efficacy of an antisense compound (AEG 35156) targeted to the X-linked inhibitor of apoptosis protein XIAP. Br J Cancer 92: $532-538$

Cummings J, Ranson M, Butt F, Moore D, Dive C (2007) Qualification of M30 and M65 ELISAs as surrogate biomarkers of cell death: long term antigen stability in cancer patient plasma. Cancer Chemother Pharmacol 60: $921-924$

Cummings J, Ward TH, Greystoke A, Ranson M, Dive C (2008) Biomarker method validation in anticancer drug development. $\mathrm{Br} J$ Pharmacology 153: $646-656$

de Haas EC, di Pietro A, Simpson KL, Meijer C, Suurmeijer AJ, Lancashire LJ, Cummings J, de Jong S, de Vries EG, Dive C, Gietema JA (2008) Clinical evaluation of M30 and M65 ELISA cell death assays as circulating biomarkers in a drug-sensitive tumor, testicular cancer. Neoplasia 10(10): $1041-1048$

Fuchs E, Weber K (1994) Intermediate filaments: structure, dynamics, function, and disease. Annu Rev Biochem 63: 345-382

Greystoke A, Cummings J, Ward T, Simpson K, Renehan A, Butt F, Moore D, Gietema J, Blackhall F, Ranson M, Hughes A, Dive C (2008) Optimisation of circulating biomarkers of cell death for routine clinical use. Ann Oncol 19: 990 - 995

Hetz H, Hoetzenecker K, Hacker S, Faybik P, Moser B, Roth G, Hoetzenecker W, Lichtenauer M, Klinger M, Krenn CG, Ankersmit H] (2007) Caspase-cleaved cytokeratin 18 and $20 \mathrm{~S}$ proteasome in liver degeneration. J Clin Lab Anal 21: 277-281

Hou JM, Greystoke A, Lancashire L, Cummings J, Ward T, Board R, Amir E, Hughes S, Krebs M, Hughes A, Ranson M, Lorigan P, Dive C, Blackhall FH (2009) Evaluation of circulating tumor cells and serological cell death biomarkers in small cell lung cancer patients undergoing chemotherapy. Am J Pathol 175: 808-816

http://www-dep.iarc.fr/.GLOBOCAN 2002. Cancer incidence, mortality and Prevalence Worldwide (2002 estimates)

Koelink PJ, Lamers CB, Hommes DW, Verspaget HW (2009) Circulating cell death products predict clinical outcome of colorectal cancer patients. BMC Cancer 9: 88

Kramer G, Erdal H, Mertens HJ, Nap M, Mauermann J, Steiner G, Marberger M, Biven K, Shoshan MC, Linder S (2004) Differentiation between cell death modes using measurements of different soluble forms of extracellular cytokeratin 18. Cancer Research 64: $1751-1756$

Kramer G, Schwarz S, Hägg M, Havelka AM, Linder S (2006) Docetaxel induces apoptosis in hormone refractory prostate carcinomas during multiple treatment cycles. Br J Cancer 94: $1592-1598$
Kummar S, Kinders R, Rubinstein L, Parchment RE, Murgo AJ, Collins J, Pickeral O, Low J, Steinberg SM, Gutierrez M, Yang S, Helman L, Wiltrout R, Tomaszewski JE, Doroshow JH (2007) Compressing drug development timelines in oncology using phase ' $0^{\prime}$ trials. Nat Cancer Rev 7: $131-139$

Leers MP, Kölgen W, Björklund V, Bergman T, Tribbick G, Persson B, Björklund B, Nap M, Jörnvall H, Schutte B (1999) Immunocytochemical detection and mapping of a cytokeratin 18 neo-epitope exposed during early apoptosis. J Pathol 187: 567-572

Lesage G, Glaser S, Ueno Y, Alvaro D, Baiocchi L, Kanno N, Phinizy JL, Francis H, Alpini G (2001) Regression of cholangiocyte proliferation after cessation of ANIT feeding is coupled with increased apoptosis. Am J Physiol Gastrointest Liver Physiol 281: $182-190$

Olofsson MH, Ueno T, Pan Y, Xu R, Cai F, van der Kuip H, Muerdter TE, Sonnenberg M, Aulitzky WE, Schwarz S, Andersson E, Shoshan MC, Havelka AM, Toi M, Linder S (2007) Cytokeratin-18 is a useful serum biomarker for early determination of response of breast carcinomas to chemotherapy. Clin Cancer Res 13: 3198-3206

Ozturk B, Coskun U, Sancak B, Yaman E, Buyukberber S, Benekli M (2009) Elevated serum levels of M30 and M65 in patients with locally advanced head and neck tumors. Int Immunopharmacol 9(5): 645-648

Roth GA, Krenn C, Brunner M, Moser B, Ploder M, Spittler A, Pelinka L, Sautner T, Wolner E, Boltz-Nitulescu G, Ankersmit HJ (2004) Elevated serum levels of epithelial cell apoptosis-specific cytokeratin 18 neoepitope M30 in critically-ill patients. Shock 22: 218-220

Schutte B, Henfling M, Kolgen W, Bouman M, Meex S, Leers MP, Nap M, Björklund V, Björklund P, Björklund B, Lane EB, Omary MB, Jörnvall $\mathrm{H}$, Ramaekers FC (2004) Keratin 8/18 breakdown and reorganization during apoptosis. Exp Cell Res 297: 11-26

Scott LC, Evans TR, Cassidy J, Harden S, Paul J, Ullah R, O'Brien V, Brown $\mathrm{R}$ (2009) Cytokeratin 18 in plasma of patients with gastrointestinal adenocarcinoma as a biomarker of tumour response. Br J Cancer 101: $410-417$

Smith RA, Bosonnet L, Ghaneh P, Raraty M, Sutton R, Campbell F, Neoptolemos JP (2008) Preoperative CA19-9 levels and lymph node ratio are independent predictors of survival in patients with resected pancreatic ductal adenocarcinoma. Dig Surg 25: 226-232

Steele NL, Plumb JA, Vidal L, Tjørnelund J, Knoblauch P, Rasmussen A, Ooi CE, Buhl-Jensen P, Brown R, Evans TR, DeBono JS (2008) A phase 1 pharmacokinetic and pharmacodynamic study of the histone deacetylase inhibitor belinostat in patients with advanced solid tumors. Clin Cancer Res 14(3): $804-810$

Sultana A, Smith CT, Cunningham D, Starling N, Neoptolemos JP, Ghaneh $P$ (2007) Meta-analyses of chemotherapy for locally advanced and metastatic pancreatic cancer. J Clin Oncol 25: 2607-2615

Ulukaya E, Yilmaztepe A, Akgoz S, Linder S, Karadag M (2007) The levels of caspase-cleaved cytokeratin 18 are elevated in serum from patients with lung cancer and helpful to predict the survival. Lung Cancer 56: 399-404

Yagmur E, Trautwein C, Leers MPG, Gressner AM, Tacke F (2007) Elevated apoptosis-associated cytokeratin 18 fragments (CK18Asp386) in serum of patients with chronic liver diseases indicate hepatic and biliary inflammation. Clin Biochem 40: 651-655

Yan L, Tonack S, Smith R, Dodd S, Jenkins RE, Kitteringham N, Greenhalf W, Ghaneh P, Neoptolemos JP, Costello E (2009) Confounding effect of obstructive jaundice in the interpretation of proteomic plasma profiling data for pancreatic cancer. J Proteome Res 8: 142-148

Yip D, Karapetis C, Strickland A, Steer CB, Goldstein D (2006) Chemotherapy and radiotherapy for inoperable advanced pancreatic cancer. Cochrane Database Syst Rev Issue 3; CD002093 